\title{
LIGHT-INDUCED DEGRADATION IN DIFFERENT p-i-n AND n-i SCHOTTKY BARRIER SOLAR CELL STRUCTURES
}

\author{
Hongyue Liu, Yeeheng Lee, T. Jamali-beh, Z. Lu, R. Collins, C. R. Wronski \\ Electronic Materials and Processing Research Laboratory, The Pennsylvania State University, \\ University Park, PA 16802
}

\begin{abstract}
The issue of the lack of reliable correlation between the characteristics of high performance cells and their intrinsic material properties is addressed in this paper. A systematic approach to the question of separating the effects of the bulk from those of the interface is described which is based on the selfconsistent analysis on a variety of both materials and solar cell properties in the annealed and degraded state. The results presented are on characteristics which include not only light I-V but also dark I-V and $Q E$ of $\mathrm{p}-\mathrm{i}-\mathrm{n}$ solar cells with different thicknesses as well as on $\mathrm{n}-\mathrm{i}-$ metal Schottky barrier cell structures. These results are discussed in terms of how the contributions of the bulk ilayers can be established and quantified in the annealed as well as the degraded states. An example is presented for such a quantitative correlation for a Schottky barrier cell structure in the annealed and degraded states.
\end{abstract}

\section{Introduction}

Improvement in the current ability to identify and quantify the mechanisms responsible for the degradation in a-Si:H based solar cells will help in guiding further improvements of their performance and stability. There have been a variety of attempts to correlate the performance of both the initial, and more recently, degraded solar cell performance with properties of the corresponding thin film materials. ${ }^{1-3}$ These correlations have been primarily based on results of light I-V's of solar cells and subgap absorption, measured by CPM or PDS on corresponding i-layer films which is used to characterize the densities of neutral dangling bonds. This assumes that the densities derived from these measurements are solely responsible for the recombination and electric field distributions in the cells. The degree of success in such correlations varies from very poor, on high performance solar cells ${ }^{2,3}$, to those which are even able to analyze the mechanisms responsible for the kinetics of degradation ${ }^{4,5}$. This issue is further complicated by the results on the high performance cells which identify the $\mathrm{p} / \mathrm{i}$ interface as being extremely important in determining both the initial as well as degraded states ${ }^{2,6}$

Because a wide range of interfaces may exist in solar cells which are fabricated under different conditions, with different materials or having different structures, it is not realistic to generalize on their role in either the initial cell characteristics or in the kinetics of degradation. However, even if the effects of the interface regions are sufficient to explain the lack of correlation for the annealed state, it is unlikely that these cells are made sufficient thin so as not to depend on their bulk properties. A more plausible explanation could be that the use of just CPM and PDS to characterize the bulk material gap states is simply inadequate. Such limited characterization does not take into account the charged defect states which have been found necessary to selfconsistently analyze photoconductivities and subgap absorption in the annealed and degraded states of a$\mathrm{Si}: \mathrm{H}^{7,8}$. It appears that improved and more reliable correlations require more detailed characterization of both materials and solar cells. This is in part due to the complex nature of the cells and in part to the many material parameters required in any quantitative correlations ${ }^{9}$. As yet no reliable analysis has been carried out on the various $\mathrm{p}$-i-n characteristics because of many "adjustable" parameters and the uncertainties introduced by the $p / i$ interface regions ${ }^{10}$. It is possible to fit light I-V's with quite different bulk material parameters by adjusting those at the interface. The reliability of these parameters can only be established by the selfconsistent analysis of a variety of material and solar cell characteristics.

This issue is addressed by the work being reported here on solar cell structures and i-layer films deposited under the same conditions. It presents the approach taken in quantifying the relative contributions of the bulk and interface region to their annealed state and to their degradation under AM1 illumination. It involves detailed characterization of the optical absorption and gap state properties in the i-layer films using $T \& R$, photoconductivity and subgap measurements (DBP) ${ }^{8}$. The parameters derived from selfconsistent fitting of the material characteristics using SAM are then further refined by the selfconsistent analysis of Schottky barrier cell characteristics with AMPS ${ }^{9}$. The parameters required include the mobility gap; band edge densities; and the energy, distribution, densities and capturecross sections of charged defects $\mathrm{D}^{+}, \mathrm{D}^{-}$as well as the neutral dangling bonds $D^{\circ}$. To take into account the complexity of the $p$-i-n solar cells, and the large number of parameters required for reliable quantitative analysis, light I-V's as well as dark I-V's and QE's with and without light bias are investigated. Similar measurements and analysis are also carried out on corresponding Schottky barrier cell structures in which there is no $\mathrm{p} / \mathrm{i}$ interface region and it is easier to directly correlate the bulk properties with their characteristics $^{11,12}$.

\section{Experimental Details}

The $p(a-S i C: H)-i(a-S i: H)-n(a-S i: H)$ and $n(a-S i: H)-$ $\mathrm{i}(\mathrm{a}-\mathrm{Si}: \mathrm{H}) / \mathrm{Ni}$ Schottky barrier cell structures were prepared in a multichamber PECVD system with i-layer from 0.4 to $\sim 1.0 \mu \mathrm{m}$ thick. The p-i-n cells fabricated on specular $\mathrm{SnO}_{2}$ substrates, so as not to have any optical enhancement 
effects, except for $J_{s c}$, had parameters close to those for similar structures reported by industry ${ }^{2}$ and the materials were what is commonly referred to as "device quality". The deposition conditions for these i-layers were: substrate temperature of $200^{\circ} \mathrm{C}$, pressure of $0.5 \mathrm{Tom}$, power density of $50 \mathrm{~mW} / \mathrm{cm}^{2}$, and flow of pure silane $15 \mathrm{sccm}$. The $\mathrm{n}-\mathrm{i}-\mathrm{Ni}$ Schottky barrier cell structures were also deposited on specular $\mathrm{SnO}_{2}$ with a $150 \AA$ thermally evaporated semitransparent top Ni contact. The device areas $\left(0.02 \mathrm{~cm}^{2}\right)$ in the Schottky barrier structures were defined by the shadow mask used in the evaporation. Same area thick $(2000 \AA) \mathrm{Cr}$ contacts were evaporated onto the n-layers of the $p-i-n$ structures and the device areas were then defined by reactive ion etching of the n-layers not covered by the $\mathrm{Cr}$. These cells were characterized by dark I-V, light I-V as well as $Q E$ measurements and the light degradation was carried out by white light from an ELH lamp with intensities of AMl to 10AMl incident through TCO.

\section{Results and Discussion}

Since it is very difficult to obtain reliable information about the contributions of the bulk properties to solar cell performance from cells with one thickness, the light I-V, dark I-V, and QE measurements were carried out on solar cells having different thicknesses. Results of such measurements are shown in Fig. 1(a), 2(a), and 3(a) for a $0.4 \mu \mathrm{m}$ thick cell and $1(b), 2(b)$ and $3(b)$ for a $0.7 \mu \mathrm{m}$ thick $p$ $i-n$ structure. The solid symbols represent the annealed state results and the open symbols represent the degraded state with 100 hours of AM1 illumination. It can be seen in Fig. l(a) and (b), that indeed the effect of the bulk can be seen in the much larger changes in the FF and $J_{s e}$ in the thick structure while the values and changes in the $V_{\infty}$ are about the same. In the thin structure, the $\mathrm{J}_{\mathrm{sc}}$, and $\mathrm{FF}$ degrade from $11.1 \mathrm{~mA} / \mathrm{cm}^{2}$, and 0.71 to $10.4 \mathrm{~mA} / \mathrm{cm}^{2}$, and 0.55 respectively. For the thick cell, the $\mathrm{J}_{\mathrm{sc}}$, and $\mathrm{FF}$ degrade from $12.1 \mathrm{~mA} / \mathrm{cm}^{2}$, and 0.60 in the annealed sate to $0.78 \mathrm{~V}, 10.6 \mathrm{~mA} / \mathrm{cm}^{2}$, and 0.46 , respectively. Because there is no optical enhancement, the $J_{s c}$ values in the two cells are directly determined by their thicknesses and the optical absorption so the changes can be accurately modeled. This is not the case of the comparable cells deposited on textured TCO where the differences are much smaller and the currents cannot be reliably quantified in terms of optical absorption. However because of the many adjustable parameters that are required in the analysis of light I-V's, reasonably good fits can still be made with different material and interface parameters. It is therefore necessary to measure and selfconsistently fit other solar cell characteristics.

The results for the far forward dark I-V's are shown in Figs. 2(a) and (b). Again it can be seen that degradation in the $0.4 \mu \mathrm{m}$ thick cells is significantly smaller than in the $0.7 \mu \mathrm{m}$ thick cell and the increased contribution of the thicker i-layer can be seen. This is also the case for the $\mathrm{QE}$ results, measured without light bias, which are shown in Figs. 3(a) and $3(\mathrm{~b})$ for the $0.4 \mu \mathrm{m}$ and $0.7 \mu \mathrm{m}$ thick cells, respectively. Because in the case of the $0.4 \mu \mathrm{m}$ cell both characteristics undergo very small changes, it is not possible to carry out reliable analysis in terms of the changes in bulk material properties. The thicker cells, on the other hand, offer characteristics in which the large effects can be more realistically analyzed, particularly the changes in the far forward bias current and the QE's, which are not masked by optical enhancement. It is clear that even with well characterized materials, quantitative analysis should be carried out first on thick cells $(>0.5 \mu \mathrm{m})$ where the light induced changes in the bulk have a much larger effect on all of the cell characteristics.

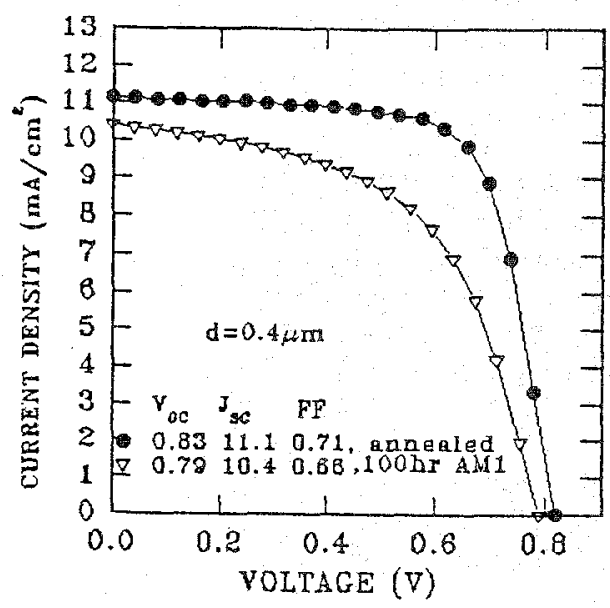

Fig. l(a) Light I-V's for a $0.4 \mu m$ thick p-i-n cell structure in the annealed and degraded state after 100 hours of AM1 light soaking

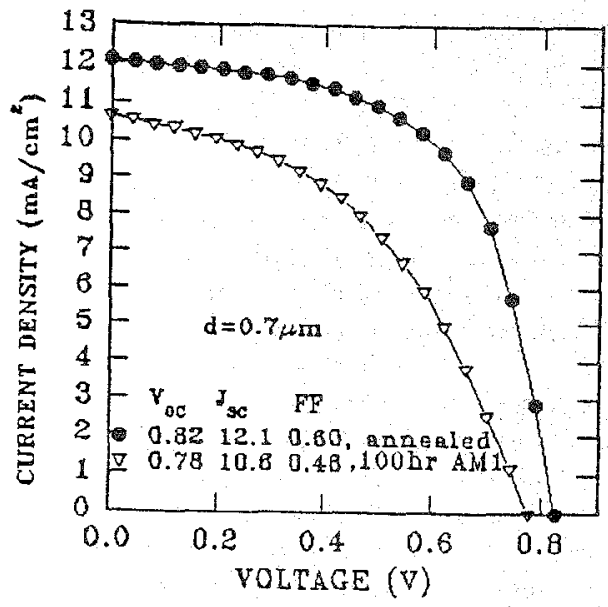

Fig. 1(b) Light I-V's for a $0.7 \mu \mathrm{m}$ thick $\mathrm{p}-\mathrm{i}-\mathrm{n}$ cell structure in the annealed and degraded state after 100 hours of AM1 light soaking 


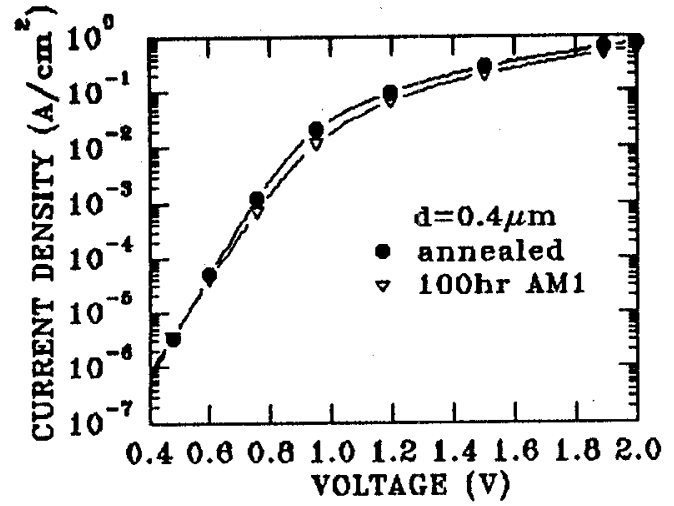

Fig. 2(a) Far forward dark I-V's for a $0.4 \mu \mathrm{m}$ and a $0.7 \mu \mathrm{m}$ thick $p-i-n$ cell structures in the annealed and degraded states after 100 hours of AM1 light soaking

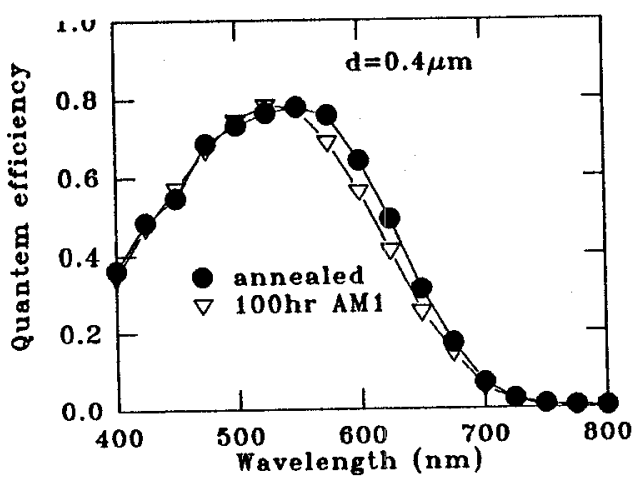

Fig. 3(a). QE's without light bias for a $0.4 \mu \mathrm{m}$ and a $0.7 \mu \mathrm{m}$ thick $p-i-n$ cell structures in the annealed and degraded states after 100 hours of AM1 light soaking

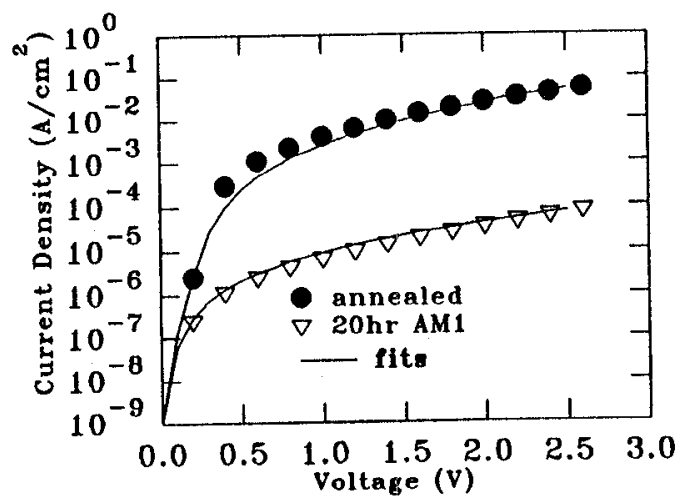

Fig. 4. Forward dark I-V's for a $1.0 \mu \mathrm{m}$ thick ni-Ni Schottky barrier structure in the annealed and degraded states. See text for details.

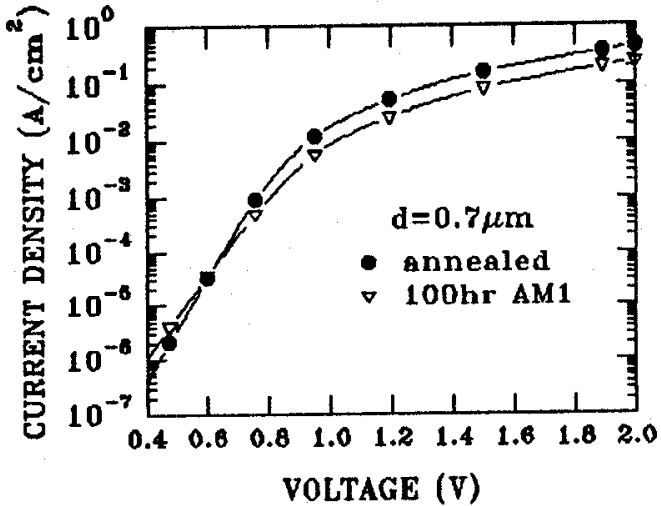

Fig. 2(b). Far forward dark 1-V's for a $0.7 \mu \mathrm{m}$ and a $0.7 \mu \mathrm{m}$ thick p-i-n cell structures in the annealed and degraded states after 100 hours of AM1 light soaking

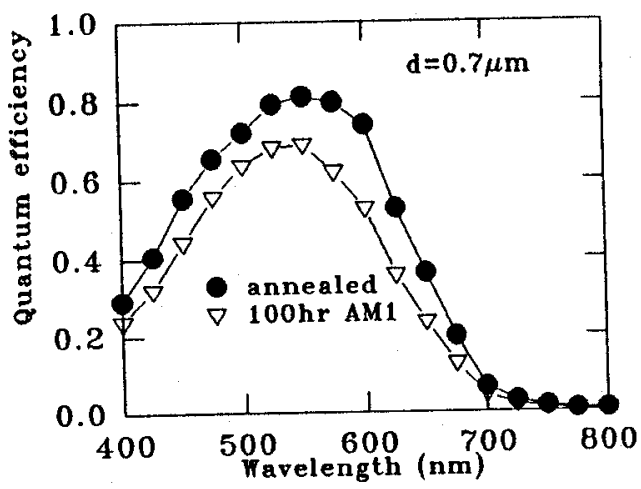

Fig. 3(b). QE's without light bias for a $0.7 \mu \mathrm{m}$ and a $0.7 \mu \mathrm{m}$ thick $p$-i-n cell structures in the annealed and degraded states after 100 hours of AM1 light soaking

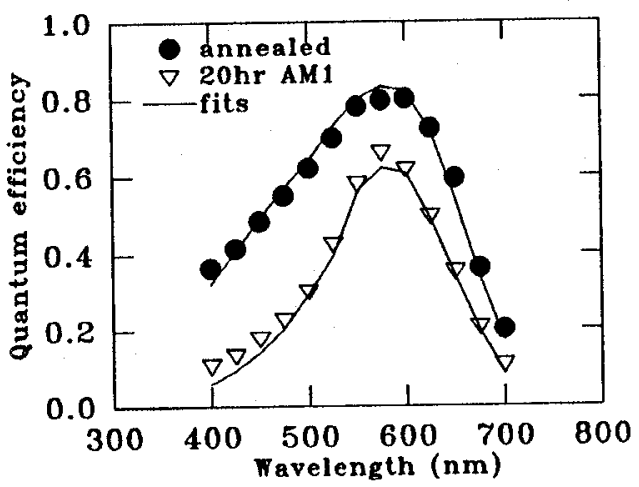

Fig. 5. QE's wihout light bias for a $1.0 \mu \mathrm{m}$ thick $\mathrm{n}$-i-Ni Schottky barrier structure in the annealed and degraded states. See text for details. 
Metal Schottky barrier solar cell structures on the other hand offer a much more direct method for correlating the properties of thin film materials with device characteristics and are particularly useful in further analyzing the bulk material properties in both the annealed and degraded states. Both the dark $\mathrm{I}-\mathrm{V}$ and $\mathrm{QE}$ are very sensitive to the bulk, and in particular to its light induced changes. So they are very useful in "fine-tuning" of the thin film material parameters for the self-consistent modeling of p-i-n cells with AMPS. Examples of this are illustrated in Figs 4 and 5, where the dark I-V's and QE's are shown in the annealed state and after degradation for 20 hours with AM1 illumination through the TCO. The symbols are experimental results and the solid lines are fits obtained with the charge defect distribution which was also used in the analysis of the thin film results. In this case the densities of charged defects increase from $5 \times 10^{15} / \mathrm{cm}^{3}$ to $7 \times 10^{15} / \mathrm{cm}^{3}$ and the neutral dangling bond defects from $5 \times 10^{15} / \mathrm{cm}^{3}$ to $1 \times 10^{17} / \mathrm{cm}^{3}$. It is important to note here that there is a three orders of magnitude change in the dark I-V of Fig. 4 which is more than one hundred times bigger than seen in Fig. 2(a) for the $p-i-n$ and can still be fitted with these distributions of gap states. In addition, similar changes in dark I-V and QE characteristics of Schottky barrier solar cell structures are observed after light soaking even with $\sim 0.4 \mu \mathrm{m}$ thick structures.

\section{Conclusions}

This paper describes an approach which may overcome the lack of correlation reported between the results on high performance cells in the annealed and soaked states with the corresponding properties of the intrinsic materials ${ }^{1,2}$

. It takes into account both the inadequacy of characterizing the gap states only with CPM measurements as well as the complexity of such solar cells and points to the need for such studies on $n^{+}(\mu \mathrm{c} \mathrm{Si})-\mathrm{i}(\mathrm{a}-\mathrm{Si}: \mathrm{H})-\mathrm{p}(\mu \mathrm{c} \mathrm{Si})$. Results are presented of dark I-V and QE measurements on different $\mathrm{p}-\mathrm{i}-\mathrm{n}$ and Schottky barrier cell structures which indicate how the contributions of the bulk i-layers can be established and more reliably quantified than those just from light I-V's. Example is presented of such a quantitative correlation for a Schottky barrier cell in both the annealed and degraded states which is achieved with analysis using parameters derived from a detailed characterization of the corresponding film. Because this selfconsistantency was achieved with gap states consisting of both the neutral and charged defects, it is therefore surprising that even in the more complicated $\mathrm{p}-\mathrm{i}-\mathrm{n}$ cell structures, using only $D^{\circ}$ states as fitting parameters, sufficiently good quantitative agreement was achieved to allow the characterization of the mechanisms responsible for degradation ${ }^{4,5}$.

\section{Acknowledgments}

The authors wish to thank Drs. J. Yang and S. Guha of USSC, Dr. L. Yang of Solarex and Dr. F. A. Rubinelli for many helpful discussions. This work is sponsored by National Renewable Energy Laboratory
(NREL) under subcontract number XAN-4-14418-03, Electric Power Research Institute (EPRI) Thin Film Solar Cell Program, and New Energy Development Organization (NEDO) International Joint Research Grant.

\section{References}

[1] X. Xu, J. Yang, and S. Guha, Appl. Phys. Lett. 62(12), 1993, p1399

[2] K. Vasanth, M. Nakata, S. Wagner and M. Bennett, Mat. Res. Soc. Symp. Proc. Vol 297, 1993, p827

[3] J. Yang, X.. Xu, S. Guha, Mat. Res. Soc. Symp. Proc. Vol 336, 1994, p687

[4] K. Vasanth, S. Wagner, D. Caputo, M. Bennett, Record of First WCPEC, Dec 5-9, 1994; Hawaii, p488 (IEEE, NY, 1995)

[5] D. Caputo, M. Maggi. G. Masini, F. Palma, K. Vananth, Mat. Res. Soc. Symp. Proc. Vol 377, 1995, p669

[6] B. Rech, C. Beneking, H. Wagner, Record of First WCPEC, Dec 5-9, 1994; Hawaii, p472 (IEEE, NY, 1995)

[7] M. Gunes and C. R. Wronski, Appl.Phys. Lett. 61, 678 (1992)

[8] Lihong Jiao, H. Liu, S. Semoushina, Y.Lee, and C. R. Wronski, Record of the 25th IEEE Photovoltaic Specialists Conference, to be published.

[9] J. K. Arch, F. A. Rubinelli, J. Y Hou and S. J. Fonash, J. Appl. Phys. 69, (1991), p7057

[10] R. Collins, et al, Record of 25th IEEE Photovoltaic Specialists Conference, to be published

[11] Mehmet Gunes, H. Liu, C. M. Fortmann, C. R. Wronski, Record of First WCPEC, Dec 5-9, 1994; Hawaii, p512 (IEEE, NY, 1995)

[12] Hongyue Liu, L. Jiao, S. Semoushikina, C. R. Wronski, J. Non-cryst. (in press) 